\title{
Idiopathic CD4+ T-cell Lymphocytopenia: Report of a Case 11 Years after Diagnosis
}

\author{
Noel Lorenzo Villalba ${ }^{1}$, Abrar-Ahmad Zulfiqar ${ }^{1}$, Yasmine Maouche ${ }^{1}$, Zaida Cordoba Sosa ${ }^{2}$, Maria Belen Alonso Ortiz ${ }^{3}$, Emmanuel Andres ${ }^{1}$ \\ ${ }^{1}$ Service de Médecine Interne, Diabète et Maladies Métaboliques, Hôpitaux Universitaires de Strasbourg, France \\ ${ }^{2}$ Internal Medicine Department, Fuerteventura General Hospital, Fuerteventura, Spain \\ ${ }^{3}$ Internal Medicine Department, Dr Negrin University Hospital, Gran Canaria, Spain
}

\section{Doi: 10.12890/2020_001589 - European Journal of Case Reports in Internal Medicine - ๑ EFIM 2020}

Received: $20 / 02 / 2020$

Accepted: 03/03/2020

Published: $30 / 03 / 2020$

How to cite this article: Lorenzo Villalba N, Zulfiqar AA Maouche Y, Cordoba Sosa Z, Alonso Ortiz MB, Andres E. Idiopathic CD4+ T-cell lymphocytopenia: report of a case 11 years after diagnosis. EJCRIM 2020;7: doi:10.12890/2020_001589.

Conflicts of Interests: The Authors declare that there are no competing interests.

This article is licensed under a Commons Attribution Non-Commercial 4.0 License

\section{ABSTRACT}

We report the case of a 23-year-old woman evaluated for asthenia and lymphocytopenia. Clinical examination was unremarkable but laboratory tests showed the presence of CD4 lymphocytopenia. Secondary causes of CD4 lymphocytopenia were ruled out and a previous diagnosis of idiopathic CD4+ T-cell lymphocytopenia was retained. CD4 lymphocytopenia has persisted for 11 years now but the patient has been clinically asymptomatic.

\section{LEARNING POINTS}

- CD4+ T-cell lymphocytopenia needs to be meticulously evaluated and secondary causes ruled out.

- The patient has been clinically asymptomatic for 11 years.

- Measurement of CD4 subsets twice yearly seems to be appropriate.

\section{KEYWORDS}

Lymphocytopenia, HIV, lymphocyte immunophenotyping

\section{INTRODUCTION}

Idiopathic CD4+ T-cell lymphocytopenia (ICL) is a rare clinical syndrome defined by a persistent unexplained deficit of circulating CD4+ T-cells in the absence of HIV infection or any other cause of immunodeficiency. For a diagnosis, CD4+ T-cell counts should be below 300 cells/ $\mu$ l or less than $20 \%$ of total lymphocytes in repeated evaluations (2-3 months apart $)^{[1,2]}$. The majority of cases are in adult individuals but the condition can also affect children and adolescents.

\section{CASE DESCRIPTION}

A 23-year-old white woman was evaluated in 2008 at an internal medicine outpatient clinic for asthenia and lymphocytopenia (722/ $\left.\mathrm{mm}^{3}\right)$. In 2019, the patient referred mild asthenia without any other complaint. Her medical history was unremarkable. She takes no medications or supplements and has no allergies. Alcohol intake or illicit drug consumption were not reported. Her family history was irrelevant.

Physical examination revealed a woman appearing to be well. The patient's vital signs were within normal limits. Her temperature was $\left(37^{\circ} \mathrm{C}\right)$, heart rate was 88 beats/min, blood pressure was $120 / 80 \mathrm{mmHg}$ and respiratory rate was 20 breaths/min. The lungs were clear to auscultation, and the heart sounds were normal and without murmur, rub or gallop. The abdomen was soft, non-tender and non-distended, with normal active bowel sounds and no hepatosplenomegaly. No enlarged lymph nodes were noted. 
Results of a complete blood cell count showed: haemoglobin $11.7 \mathrm{~g} / \mathrm{dl}$, mean corpuscular volume $83 \mathrm{fl}$, and leucocytes $3.7 \mathrm{~g} / \mathrm{l}$ (NV: 4-10) with isolated lymphocytopenia $625 / \mathrm{mm}^{3}$ (NV: 1,500-4,000) and a normal platelet count and erythrocyte sedimentation rate. A second blood cell count 1 month later in the same laboratory also showed isolated lymphocytopenia $\left(625 / \mathrm{mm}^{3}\right)$. Coagulation, thyroid, liver and renal tests were within the normal range. An HIV test was negative on two occasions.

Serum protein electrophoresis with immunofixation electrophoresis was normal. Vitamin B12 and folic acid levels were normal. Serology for hepatitis B and C, CMV, EBV, herpes virus, parvovirus B19 and syphilis was negative. Mycoplasma, rickettsia and Borrelia burgdorferi tests were negative.

Angiotensin converting enzyme was within the normal range. Quantiferon was negative. Rheumatoid factor, antinuclear antibodies, antiSSA/SSB antibodies, antineutrophil cytoplasmic antibodies and antimyeloperoxidase antibodies were negative. Total complements as well as C3 and C4 fractions were within the normal range. Beta-2 microglobulin was normal. Serum immunoglobulins (IgG, IgA and IgM) were normal. Repeated oral and vaginal thrush testing was negative. Chest $x$-rays and an abdominal ultrasound were normal.

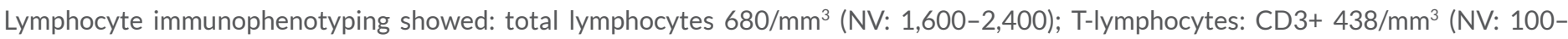
1,700), 64.4\% (NV: 67-76), CD4+321/mm³ (NV: 1,600-2,400), CD3+/CD4+ 210/mm³ (NV: 700-1,100), 30.9\% (NV: 38-46), and CD + / CD8+ 155/mm³ (NV: 500-900), 22.8\% (NV: 31-40).

A second evaluation of the lymphocyte populations showed: CD45+ 100\%; B-lymphocytes: CD19+16\% (NV: 11-16), 151×10\%/I (NV: 200-

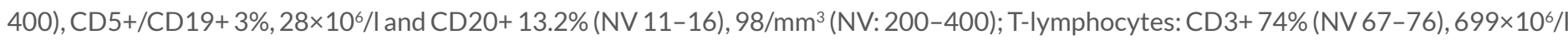
(NV: 1,100-1,700), CD5+ 74\%, 699×10\%/l, CD3+/CD4+ 43\% (NV: 38-46), 406×10\%/l (NV: 700-1,100), CD3+/CD8+ 26\% (NV 31-40), $245 \times 10^{6} / \mathrm{I}(\mathrm{NV}: 500-900), \mathrm{CD} 4+/ \mathrm{CD} 8+2 \%, 19 \times 10^{6} / \mathrm{I}$ and CD4/CD8 1.65; CD3/NK: CD56+/CD16+26\%, 245×10\%/l, CD3+/CD56+Cdd16+ 14\%, 132×10 $/$ /, CD3-/CD56+CD16+ 12\%, 113×10\%/l, and CD 56+/Cd3- 79/mm³ (NV: 200-400). Study of negative controls showed: IgG1 FITC (BD) control at $0 \%$, IgG1 PE (BD) control at $0 \%$, and IgG2a FITC (BD) control at $0 \%$. These results were consistent with an isolated CD4+ T-cell lymphocytopenia in the absence of an inflammatory, immunological or associated infectious condition.

The diagnosis of ICL was retained and the patient was clinically and biologically followed up. There are no guidelines for monitoring patients with ICL. Since diagnosis, the patient has been clinically asymptomatic and measurement of CD4 subsets twice yearly was considered to be appropriate (Table 1).

\begin{tabular}{|l|l|l|}
\hline \multicolumn{1}{|c|}{ Year } & \multicolumn{2}{c|}{ Absolute CD4 } \\
\hline 2008 & $321 / \mathrm{mm} 3$ & $300 / \mathrm{mm} 3$ \\
\hline 2009 & $290 / \mathrm{mm} 3$ & $301 / \mathrm{mm} 3$ \\
\hline 2010 & $300 / \mathrm{mm} 3$ & $285 / \mathrm{mm} 3$ \\
\hline 2011 & $320 / \mathrm{mm} 3$ & $295 / \mathrm{mm} 3$ \\
\hline 2012 & $299 / \mathrm{mm} 3$ & $298 / \mathrm{mm} 3$ \\
\hline 2013 & $280 / \mathrm{mm} 3$ & $299 / \mathrm{mm} 3$ \\
\hline 2014 & $300 / \mathrm{mm} 3$ & $287 / \mathrm{mm} 3$ \\
\hline 2015 & $291 / \mathrm{mm} 3$ & $290 / \mathrm{mm} 3$ \\
\hline 2016 & $300 / \mathrm{mm} 3$ & $301 / \mathrm{mm} 3$ \\
\hline 2017 & $281 / \mathrm{mm} 3$ & $299 / \mathrm{mm} 3$ \\
\hline 2018 & $305 / \mathrm{mm} 3$ & $289 / \mathrm{mm} 3$ \\
\hline 2019 & $295 / \mathrm{mm} 3$ & $301 / \mathrm{mm} 3$ \\
\hline
\end{tabular}

\section{DISCUSSION}

Patients can be asymptomatic as in the case reported or present with opportunistic infections, malignancies and/or autoimmune disorders ${ }^{[1]}$. Immune dysregulation is considered to be responsible for the clinical manifestations in this syndrome and the degree of lymphopenia predicts disease. Opportunistic infections are seen when the absolute CD4 cell count drops below 200 cells/mm. Patients with severe immunosuppression are clinically indistinguishable from those with advanced HIV infection. 
A wide variety of infections has been reported, including, for example, persistent genital infection with human papilloma virus (HPV), varicella-zoster virus, cryptococcal meningitis, mycobacterial infections, Fusobacterium nucleatum, Salmonella typhimurium causing sepsislike presentations, Actinomycosis species, Rhodococcus equi and cytomegalovirus retinitis ${ }^{[3,4]}$. ICL rarely presents with recurrent bacterial infections even though this clinical picture has more often been described in children.

Malignancies such as lymphomas are quite prevalent in these patients ${ }^{[5]}$ and Kaposi sarcoma of the digestive tract or skin has also been described. Autoimmune disorders, especially entities involving the skin and mucous membranes, have also been reported. Of note, they can present either before or after the diagnosis of ICL is made. The spectrum is wide and may include, for example, idiopathic thrombocytopenic purpura and systemic lupus erythematosus ${ }^{[3]}$.

$\mathrm{ICL}$ is a diagnosis of exclusion; no infections or immunological abnormalities should be present, as in our case. The differential diagnosis should include acute or chronic retroviral infections, sarcoidosis, common variable immunodeficiency, congenital immunodeficiencies, chemotherapy immunosuppression, cancer, acute respiratory distress syndrome and autoimmune disorders.

The prognosis is variable and depends on the severity of immunosuppression, with most severe infections occurring at diagnosis or soon thereafter. There is no consensus concerning monitoring and treatment, but management of ICL should be focused on the treatment of associated conditions.

Régent et al. prospectively followed 40 patients from 1991 to 2012. In their study, infections developed in 25 patients at diagnosis or during follow-up. At the time of diagnosis, the mean CD4 T-cell count was $127 / \mathrm{mm}^{3}$ (significantly below our patient's initial CD4 count). The authors also reported that at the end of follow-up all but one of the patients with available CD4 T-cell counts showed persistent CD4 T-cell counts. They also highlighted that an initial CD4 T-cell count $<150 / \mathrm{mm}^{3}$ or a low NK cell count $\left(<100 / \mathrm{mm}^{3}\right)$ were related to a poor prognosis ${ }^{[5]}$. Even though fortuitous detection of asymptomatic patients with ICL has been reported, probably only a minority of patients remain asymptomatic as in the case presented.

\section{CONCLUSIONS}

ICL is a rare condition and it is a diagnosis of exclusion. CD4 cells stabilize at a low level rather than continuing to fall as in HIV infection.

\section{REFERENCES}

1. Gholamin M, Bazi A, Abbaszadegan MR. Idiopathic lymphocytopenia. Curr Opin Hematol 2015;22:46.

2. Ho DD, Cao Y, Zhu T, et al. Idiopathic CD4+ T-lymphocytopenia--immunodeficiency without evidence of HIV infection. N Engl J Med 1993;328:380.

3. Ahmad DS, Esmadi M, Steinmann WC. Idiopathic CD4 lymphocytopenia: spectrum of opportunistic infections, malignancies, and autoimmune diseases. Avicenna J Med 2013;3:37.

4. Longo F, Hébuterne X, Michiels JF, et al. [Multifocal MALT lymphoma and acute cytomegalovirus gastritis revealing CD4 lymphopenia without HIV infection]. Gastroenterol Clin Biol 1999;23:132.

5. Régent A, Autran B, Carcelain G, et al; French Idiopathic CD4 T Lymphocytopenia Study Group. Idiopathic CD4 lymphocytopenia: clinical and immunologic characteristics and follow-up of 40 patients. Medicine (Baltimore) 2014;93(2):61-72. 